\title{
Energy level determination of purine containing blue light emitting organic compounds
}

\author{
Raitis Grzibovskis ${ }^{\mathrm{a} *}$, Aivars Vembris ${ }^{\mathrm{a}}$, Armands Sebris ${ }^{\mathrm{b}}$, Zigfrids Kapilinskis $^{\mathrm{b}}$, Maris Turks ${ }^{\mathrm{b}}$ \\ ${ }^{a}$ Institute of Solid state physics, University of Latvia, Riga, Latvia; ${ }^{b}$ Faculty of Materials Science and \\ Applied Chemistry, Riga Technical University, Riga, Latvia
}

\begin{abstract}
The organic light emitting diodes (OLED) have found their applications in mobile and TV screens. Till now commercially available diodes are made by expensive thermal evaporation in a vacuum. The costs of OLED fabrication could be decreased by applying low-cost wet casting methods, for example, spin-coating. In this work, we have studied a group of blue light emitting purine derivatives which could potentially be used in OLEDs. The advantage of these compounds is their ability to form amorphous thin films from solutions. All the thin films were prepared by the spincoating method from chloroform solution on ITO glass. To evaluate the possibility to use purine derivatives in the creation of OLEDs, energy level values were obtained. Ionization energy was determined using photoelectron yield spectroscopy. The gap between ionization energy and electron affinity was determined using photoconductivity measurements. Electron affinity $\left(E_{a}\right)$ then was calculated as a difference between ionization energy $(I)$ and photoconductivity threshold value $\left(E_{t h}\right)$.
\end{abstract}

Changes in the energy level values depending on the position of electron acceptor group, the type of the attached substitute group, and the size of the bulky group will be discussed.

The abstract summarizes key findings in the paper. It is a paragraph of 250 words or less. For the keywords, select up to 8 key terms for a search on your manuscript's subject.

Keywords: OLED, energy levels, photoelectron emission, photoconductivity, purine

\section{INTRODUCTION}

The organic light emitting diodes (OLED) are widely used in mobile and TV screens. Till now most of the commercially available diodes are made by the expensive thermal evaporation in a vacuum. The costs of OLED fabrication could be decreased by applying low-cost wet casting methods, for example, spin-coating, inkjet printing or roll-to-roll technologies. Red and green OLEDs are well developed while there still are problems with blue OLEDs- unsatisfactory long term stability, high driving voltage, .... . Energy level compatibility between the used materials is a crucial parameter to reach high efficiency of OLEDs. It is especially important in the case of materials for blue OLEDs. As the gap between ionization energy and electron affinity is relatively large $(\sim 3 \mathrm{eV})$, one of two situations can occur: electron affinity level can be relatively high (close to vacuum level), creating barrier for electron transport, or ionization energy can be low (far away from vacuum level), creating barrier for hole transport. Depending on all the other used materials, energy levels of active material have to be "tuned" to minimize potential barriers.

In this work, we have studied a group of blue light emitting purine derivatives which could potentially be used in OLEDs. The advantage of these compounds is their ability to form amorphous thin films from solutions, which allows using wet casting methods. To evaluate the possibility to use these compounds in the creation of OLEDs there are several parameters to study. Here we have focused on the determination of energy levels of purine containing molecules. Energy level values depending on molecule structure have been examined. 


\section{EXPERIMENTAL}

\subsection{Studied compounds}

In this work, three groups of organic compounds containing purine were studied.

In the first group, all of the molecules 1-8 contain triphenylpentyl-9H-purine and have two groups which switch places in molecules 1-4 and 5-8 Compounds 1-4 have phenyl-1H-1,2,3-triazole at the $2^{\text {nd }}$ position in the purine and piperidine at $6^{\text {th }}$ position. In the molecules 5-8 these two groups switch places. Additionally, four different substitute groups are added to the phenyl-1H-1,2,3-triazole: dimethylamine group (molecules 1 and 5), cyano group (molecules 2 and 6), metoxy group (molecules 3 and 7), and hydrogen atom (molecules 4 and 8).

In the second group, molecules 9-14 contain triphenylhexyl-9H-purine and again two groups which switch places between $2^{\text {nd }}$ and $6^{\text {th }}$ position in the purine. These two groups are $1 \mathrm{H}-1,2,3$-triazole and piperidine. Again, there are substitute groups added to the 1H-1,2,3-triazole: phenyl group (molecules 9 and 12), hydroxyl group (molecules 10 and 13) and (3,3,3-triphenylpropyonyl)hydroxymethyl group (molecules 11 and 14).

Third group of molecules is 2-(4-(4-cyanophenyl)-1H-1,2,3-triazole)-6-piperidine-9H-purine with attached four different "tails" containing bulky triphenyl group: 2-ethoxy 3,3,3-triphenylpropanoate (molecule 15), 2-ethoxy 4,4,4triphenylbutanoate (molecule 16), (poly ethylene glycol) 4 3,3,3-triphenylpropanoate (molecule 17), and (poly ethylene glycol) 4 4,4,4-triphenylbutanoate (molecule 18).

All of the molecules are shown in Figure 1. 

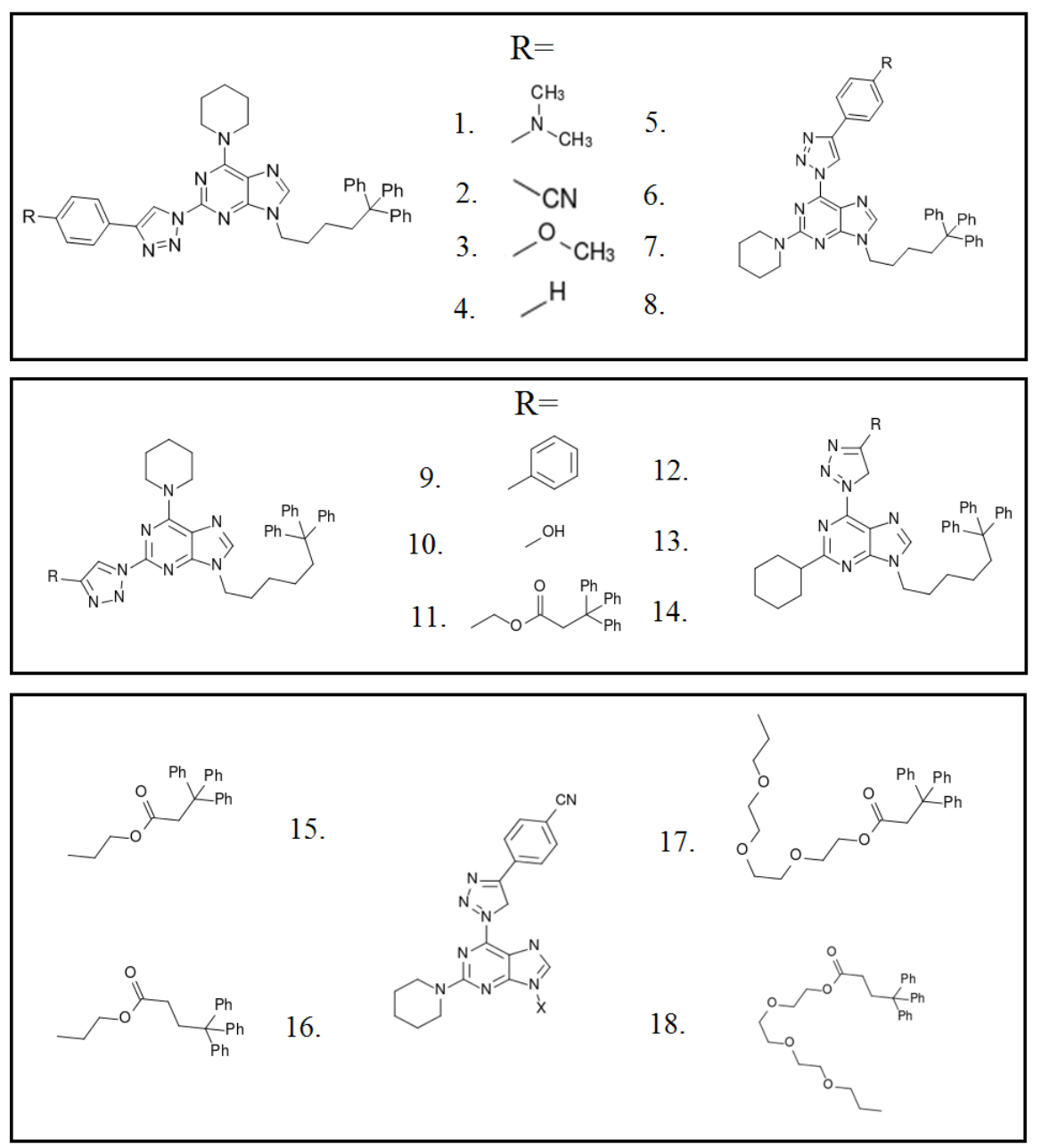

Figure 1. Studied compounds.

\subsection{Sample preparation}

Samples for photoelectron emission and photoconductivity measurements were prepared on the indium tin oxide (ITO) covered glass substrates. ITO was etched away using hydrochloric acid $(\mathrm{HCl})$ and zinc $(\mathrm{Zn})$ to obtain $1 \mathrm{~cm}$ wide electrode. Substrates were ultrasonically cleaned before the use.

Samples were made from chloroform solution with the concentration of $40 \mathrm{mg} / \mathrm{ml}$ using Laurell WS-650Sx-GNPP/Lite spin-coater. Spin-coating parameters were: rotation speed of $500 \mathrm{rpm}$, acceleration of $500 \mathrm{rpm} / \mathrm{s}$, spinning time of $50 \mathrm{~s}$. Samples were dried on a hot plate at $70^{\circ} \mathrm{C}$ for 15 min. The thickness of these samples was in the range from $400 \mathrm{~nm}$ to $600 \mathrm{~nm}$. The thickness of these samples was measured using Veeco Dektak 150 surface profilometer.

After photoelectron emission measurements, $25 \mathrm{~nm}$ thick aluminum ( $\mathrm{Al})$ electrodes were deposited on the samples using Edwards Auto 360 thermal evaporation system. In this way "sandwich" type samples (Al/ organic compound/ ITO) were created.

Samples from solution with lower concentration $(\sim 15 \mathrm{mg} / \mathrm{ml})$ were made for absorption measurements. Spin-coating parameters were the same as previously described. These samples were made on quartz substrates. Absorption spectra were measured using Ocean Optics HR4000CG-UV-NIR spectrometer. 


\subsection{Photoemission yield spectroscopy measurements}

Ionization energy of the studied compounds was obtained using self-built photoemission yield spectroscopy system. Measurements were done in vacuum $\left(\sim 10^{-5} \mathrm{mBar}\right)$ to avoid the influence of the atmosphere. ENERGETIQ Laser Driven Light Source (LDLS EQ-99) was used as a source of ultraviolet radiation. Incident photon energy was changed by diffraction grating monochromator MYM-1 with the step of $0.05 \mathrm{eV}$ in the spectral range between $4 \mathrm{eV}$ and $6.5 \mathrm{eV}$. Keithley 617 electrometer was used as the equipment to measure the number of emitted electrons as well as a voltage source. Applying a voltage of $50 \mathrm{~V}$ improved the signal to noise ratio by one order of magnitude. At the distance of $2 \mathrm{~cm}$ from the sample there was an electrode which collected the emitted electrons.

Photoemission yield $\mathrm{Y}(h v)$ was calculated as

$$
Y(h v)=\frac{I(h v)}{P(h v)}
$$

where $\mathrm{I}(h v)$ is the number of emitted electrons, $\mathrm{P}(h v)$ is the number of incident photons with the energy of hv. In threshold region a relation between photoemission yield $\mathrm{Y}(h v)$ and ionization energy $\mathrm{E}_{\text {ioniz }}$ can be expressed as a power law

$$
Y(h v)=\alpha\left(h v-E_{\text {ioniz }}\right)^{n}
$$

where $\alpha$ is constant showing amplitude of the signal and $n=1 \ldots 3$ depending on studied materials ${ }^{1} . \mathrm{n}=2$ is used in the case of metals ${ }^{2,3}$, while $n=2.5$ or $n=3$ is used in the case of semiconductors ${ }^{4-6}$. We used $n=2.5$ as it gave better approximation than $n=3$.

\subsection{Photoconductivity}

Setup of the photoconductivity measurements was similar to the setup for photoelectron emission measurements. The difference was that in photoconductivity measurements we used "sandwich" type (ITO/ studied compound/ Al) samples and there was no electrode $2 \mathrm{~cm}$ away from the sample to collect electrons. Measurement range was from $500 \mathrm{~nm}$ to $300 \mathrm{~nm}$ with a step of $5 \mathrm{~nm}$.

Photoconductivity quantum efficiency can be calculated as

$$
\beta(h v)=\frac{j(h v)}{e \cdot k(h v) \cdot i(h v) \cdot g(h v)}
$$

where $\mathrm{j}(\mathrm{h} v)$ is photocurrent density $\left(\mathrm{A} / \mathrm{cm}^{2}\right)$, e is elementary charge, $\mathrm{k}(\mathrm{h} v)$ is the transmittance of the semitransparent electrode, $\mathrm{i}(\mathrm{h} v)$ is the intensity of light $\left(\mathrm{phot} . / \mathrm{cm}^{2} \cdot \mathrm{s}\right)$ and $\mathrm{g}(\mathrm{h} v)$ is coefficient describing the portion of light absorbed in the thin film.

Similarly as in the photoelectron emission, in the threshold region a power law can be used:

$$
\beta(h v)=x\left(h v-E_{t h}\right)^{2.5}
$$

where $\mathrm{x}$ is the amplitude of the signal, $\mathrm{E}_{\mathrm{th}}$ is photoconductivity threshold energy.

Photoconductivity threshold energy is considered to be the difference between ionization energy and electron affinity of the studied compound. From this, we can calculate electron affinity value:

$$
E_{a}=I-E_{t h}
$$

\section{RESULTS AND DISCUSSION}



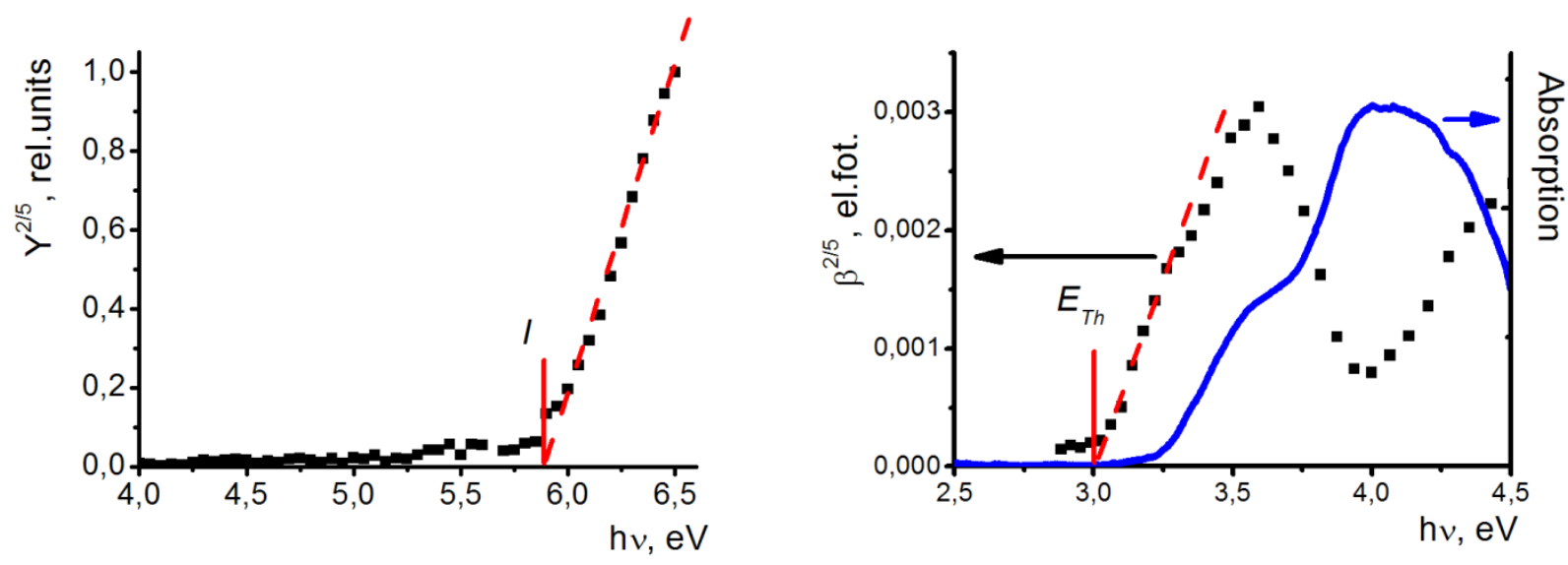

Figure 2. Example of a) photoemission yield spectrum and ionization energy determination, b) photoconductivity spectrum and photoconductivity threshold energy determination.

In the Fig. 2 we have shown examples of ionization energy and photoconductivity threshold energy determination. In both cases, no notable signal is observed under the threshold energy and then there is rapid increase in the signal. After reaching photon energy, which is considered to be the ionization energy of studied material, the photoemission yield is constantly growing. In the photoconductivity spectrum after growth of quantum efficiency there is a rapid decrease which coincides with the absorption maximum of the compound. This shows that photoconductivity is not an interface effect but rather comes from the bulk of the sample.

Table 1. Energy level values of the studied compounds.

\begin{tabular}{|l|l|l|l|l|l|l|l|}
\hline No. & $\mathrm{I}, \mathrm{eV}$ & $\mathrm{E}_{\mathrm{th}}, \mathrm{eV}$ & $\mathrm{E}_{\mathrm{a}}, \mathrm{eV}$ & No. & $\mathrm{I}, \mathrm{eV}$ & $\mathrm{E}_{\mathrm{th}}, \mathrm{eV}$ & $\mathrm{E}_{\mathrm{a}}, \mathrm{eV}$ \\
\hline 1 & 5.25 & 3.07 & 2.18 & 5 & 5.25 & 2.78 & 2.47 \\
\hline 2 & 5.76 & 3.45 & 2.31 & 6 & 5.80 & 2.95 & 2.85 \\
\hline 3 & 5.85 & 3.26 & 2.59 & 7 & 5.86 & 2.79 & 3.07 \\
\hline 4 & 6.04 & 3.15 & 2.89 & 8 & 5.90 & 2.75 & 3.15 \\
\hline
\end{tabular}

\begin{tabular}{|l|l|l|l|l|l|l|l|}
\hline 9 & 5.75 & 3.21 & 2.54 & 12 & 5.81 & 2.69 & 3.12 \\
\hline 10 & 5.88 & 3.22 & 2.66 & 13 & 5.79 & 2.70 & 3.09 \\
\hline 11 & 5.93 & 3.25 & 2.68 & 14 & 5.89 & 2.75 & 3.14 \\
\hline
\end{tabular}

\begin{tabular}{|l|l|l|l|l|l|l|l|}
\hline 15 & 5.93 & 2.80 & 3.13 & 17 & 5.88 & 2.76 & 3.12 \\
\hline 16 & 5.97 & 2.77 & 3.20 & 18 & 5.79 & 2.75 & 3.04 \\
\hline
\end{tabular}

In the Table 1 we have collected energy level values of all 18 studied compounds. There are several observations and conclusions we can gather from these values. It seems that $1 \mathrm{H}-1,2,3$-triazole and piperidine group location on the purine 
fragment has little influence on the ionization energy values: ionization energy values are almost independent on the location of these two groups. Ionization energy "tuning" is achieved by the added substitute groups. The difference in the ionization energy for the compounds 1 and 4 is almost $0.8 \mathrm{eV}$ (the difference between ionization energy of compounds 5 and 8 is $0.65 \mathrm{eV}$ ). For the compounds $9-11$ and 12-14 these changes are less notable. By adding certain substitute groups to the molecule, it is possible to tune ionization energy to obtain desired value.

Switching the positions of $1 \mathrm{H}-1,2,3$-triazole and piperidine group has notable influence on the photoconductivity threshold energy (gap between ionization energy and electron affinity). The difference of $\mathrm{E}_{\text {th }}$ between molecules 1-4 and respective molecules 5-8 is around 0.40-0.50 eV. Similar $\mathrm{E}_{\mathrm{th}}$ difference of around $0.50 \mathrm{eV}$ is observed for molecules $9-11$ and respective molecules 12-14. As the ionization energy values were almost independent on the position of these groups, changes of $\mathrm{E}_{\mathrm{th}}$ directly influence the value of electron affinity. It shows that by changing the position if certain groups in the molecule we can change ("tune") electron affinity value.

Although the changes are relatively small, molecules containing shorter "tails" with attached bulky groups (compounds 15 and 16) have higher ionization energy (by absolute value) than the molecules with longer "tails" (compounds 17 and 18). The (poly ethylene glycol) $)_{4}$ group in molecules 17 and 18 is too long to disrupt the interaction between molecules efficiently. As the molecules can interact, their ionization energy becomes lower.

\section{CONCLUSIONS}

We have studied the energy level values of 18 different purine containing compounds. It has been observed that ionization energy can be changed in relatively wide range by adding different substitute groups to the molecule. Electron affinity value can be altered by changing the position of different groups in the molecule. Small changes in the ionization energy values can be achieved by altering the length of the "tail" where inactive bulky group is added.

\section{ACKNOWLEDGEMENTS}

This research is financially supported by

\section{REFERENCES}

[1] Kane, E. O., “Theory of Photoelectric Emission from Semicoductors,” Phys. Rev. 127(1), 131-141 (1962)

[2] Monjushiro, H., Watanabe, I.., Yokoyama, Y., "Ultraviolet Photoelectron Yield Spectra of Thin Gold Films Measured in Air,” Anal. Sci. 7(4), 543-547 (1991).

[3] Ow-Yang, C. W., Jia, J., Aytun, T., Zamboni, M., Turak, a., Saritas, K.., Shigesato, Y., "Work function tuning of tin-doped indium oxide electrodes with solution-processed lithium fluoride," Thin Solid Films 559, 58-63, Elsevier B.V. (2014).

[4] Kanai, K., Honda, M., Ishii, H., Ouchi, Y.., Seki, K., "Interface electronic structure between organic semiconductor film and electrode metal probed by photoelectron yield spectroscopy," Org. Electron. 13(2), 309319, Elsevier B.V. (2012).

[5] Gao, Y., "Surface analytical studies of interfaces in organic semiconductor devices," Mater. Sci. Eng. R Reports 68(3), 39-87, Elsevier B.V. (2010). 
[6] Honda, M., Kanai, K., Komatsu, K., Ouchi, Y., Ishii, H.., Seki, K., “Atmospheric effect of air, N[sub 2], O[sub 2], and water vapor on the ionization energy of titanyl phthalocyanine thin film studied by photoemission yield spectroscopy,” J. Appl. Phys. 102(10), 103704 (2007). 